\title{
Heart failure and acute renal dysfunction in the cardiorenal syndrome
}

\author{
Authors: Rajinder S Chahal, ${ }^{\mathrm{A}}$ Chukwuma A Chukwu, ${ }^{\mathrm{B}}$ Paul R Kalra ${ }^{\mathrm{C}}$ and Philip A Kalra ${ }^{\mathrm{D}}$
}

Just under 1 million people in the UK have symptomatic heart failure. Decompensated heart failure is associated with a particularly poor prognosis with in-hospital mortality at around $10 \%$. Over the last 30 years renin-angiotensinaldosterone system antagonists have been shown to have incremental benefit on improved quality of life, reduced hospitalisation and mortality rates in those with heart failure with reduced ejection fraction. Concomitant chronic kidney disease and 'acute kidney injury' are common and associated with adverse outcomes.

In patients with decompensated heart failure, congestion is a key driver of deterioration in renal function. Decongestion is fundamental to successful management. Yet it is not uncommon to see prognostically important medication (such as angiotensin converting enzyme inhibitors and mineralocorticoid antagonists) inappropriately stopped, along with under-diuresis of the patient. This leaves the patient still in a state of congestion without the prognostic medication at discharge, with resultant adverse outcome. The British Society for Heart Failure and the Renal Association have produced consensus guidance to help guide management in a more consistent fashion based on heart failure classification, whether the patient is congested and the degree of renal impairment. Early heart failure specialist review is associated with improved patient outcomes.

DOI: 10.7861/clinmed.2019-0422

\section{The burden of heart failure}

It is estimated that 920,000 people are living with heart failure in the UK. The incidence of heart failure increased by $12 \%$ from 170,727 in 2002 to 190,798 in 2014, in part due to an increase in population size. Prevalence increased by $23 \%$ over the same 12 -year period and is influenced by improved survival with the condition. ${ }^{1}$ Despite many patients having symptoms that should

Authors: ${ }^{\text {A }}$ heart failure and devices fellow, Portsmouth Hospitals NHS Trust, Portsmouth, UK; ${ }^{B}$ research fellow in nephrology, Salford Royal NHS Foundation Trust, Salford, UK; ' Consultant cardiologist, Portsmouth Hospitals NHS Trust, Portsmouth, UK and honorary

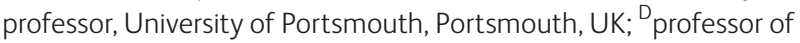
nephrology, Salford Royal NHS Foundation Trust, Salford, UK and University of Manchester, Manchester, UK have triggered an earlier assessment, up to $80 \%$ of heart failure diagnoses are made in hospital. ${ }^{2}$

In heart failure with reduced ejection fraction (HFrEF), reninangiotension-aldosterone system inhibitors (RAASi) have shown great prognostic benefits with network-meta-analysis of pooled data showing $43 \%$ reduction in mortality for those taking both an angiotensin converting enzyme inhibitor (ACEi) and a mineralocorticoid receptor antagonist (MRA), when compared to placebo. $^{3}$

\section{Key points}

Congestion is the primary driver for acute kidney injury (AKI) in decompensated heart failure and persistence of congestion is associated with worse outcomes. Decongestion with diuretics is the key to reducing symptoms and improving patient outcomes.

Although the occurrence of AKI in the patient with heart failure with reduced ejection fraction (HFrEF) infers a high-risk state, this should not routinely lead to discontinuation of reninangiotension-aldosterone system inhibitors (RAASi) therapy. RAASi are not nephrotoxic.

The cause of worsening renal function during a decompensation episode needs careful thought, particularly regarding the volume status of the patient and whether the RAASi are of prognostic benefit (eg they are for patients with HFrEF, but no evidence exists for this benefit in heart failure and preserved ejection fraction).

The consensus advice from the Renal Association and the British Society for Heart Failure gives clinicians a helpful framework to guide RAASi therapy.

Specialist care makes a difference in the acute inpatient and outpatient setting. All suspected heart failure cases, and those with unexplained acute breathlessness, should have an $\mathrm{N}$-terminal pro B-type natriuretic peptide level check and be referred for review.

The addition of RAASi before discharge improves patient outcomes. Early heart failure specialist follow-up must occur with a view to up-titration of the RAASi in HFrEF.

KEYWORDS: Heart failure, decompensation, chronic kidney disease, cardiorenal syndrome, acute kidney injury 
A combination of angiotensin receptor blocker (ARB) and neprilysin inhibitor (sacubitril valsartan) offers additional benefit beyond ACEi for patients with HFrEF. The PARADIGM-HF study showed that sacubitril valsartan significantly reduced the risks of death and hospitalisation for heart failure when compared to ACEi. ${ }^{4}$ However, this was not the case for patients with heart failure and preserved ejection fraction (PARAGON-HF study). ${ }^{5}$

Patients with heart failure are at risk of hospitalisation from decompensation. National audit data has shown that annually there are nearly 90,000 admissions in England and Wales, with an in-hospital mortality of $9.4 \% .{ }^{6}$ Despite clear evidence suggesting improved post-discharge mortality rates (at 1 year), less than half of patients with HFrEF are discharged from hospital with a combination of ACEi/ARB, beta-blocker and MRA. ${ }^{6}$

The cornerstone of managing patients hospitalised with decompensated heart failure is addressing their congestion with diuretic therapy. In this scenario, worsening renal function is frequently observed but this can then lead to suboptimal management and unnecessary discontinuation of vital medications such as RAASi.

\section{Prevalence and adverse association of chronic kidney disease and acute kidney injury in patients with heart failure}

The most prognostically important comorbidity in patients with heart failure is chronic kidney disease (CKD). The majority of patients have a chronic reduction in glomerular filtration (estimated glomerular filtration rate (eGFR) of $<60 \mathrm{~mL} / \mathrm{min} / 1.73 \mathrm{~m}^{2}$ ). CKD is more predictive of mortality than the degree of left ventricular impairment in patients with chronic heart failure (CHF). ${ }^{7}$ Close to $10 \%$ of the UK adult population have CKD. This proportion increases to over $50 \%$ in patients with $\mathrm{CHF}^{8}$. Acute deteriorations in renal function, previously referred to as worsening renal function but now as acute kidney injury (AKI), and defined as a rise in serum creatinine by more than $26.5 \mu \mathrm{mol} / \mathrm{L}(0.3 \mathrm{mg} / \mathrm{dL})$ is observed in $20-40 \%$ of patients hospitalised with acute decompensated heart failure. ${ }^{9,10}$ Baseline CKD, hypertension, diabetes, increasing age and diuretic use are predictors for the occurrence of AKI. ${ }^{11,12}$ The prognostic significance of such changes in renal function during treatment of decompensated heart failure has been debated in different studies. ${ }^{13,14}$

Pathophysiology of acute kidney injury in decompensated heart failure (cardiorenal syndrome)

Complex haemodynamic, neurohumoral, inflammatory and oxidative mechanisms underpin the development and progression of the cardiorenal syndrome (CRS). ${ }^{15,16,17}$ A reduced cardiac output and arterial filling pressure, as well as an elevated central venous pressure (CVP) due to systemic venous congestion and decreased renal perfusion, trigger a variety of neurohumoral (mal)adaptive mechanisms such as the activation of the renin-angiotensinaldosterone system (RAAS), the sympathetic nervous system (SNS) and antidiuretic hormone (arginine vasopressin) secretion. Furthermore, RAAS upregulation itself triggers a pro-inflammatory state with associated detrimental oxidative stress.

These neurohumoral responses, which are aimed at restoring perfusion to vital organs in a compromised circulatory system, can potentially create a self-perpetuating vicious cycle of fluid retention, venous congestion, tissue hypoperfusion, inflammation and oxidative stressors, which can result in further deterioration of both cardiac and renal function. ${ }^{18}$ A report detailing insights gained from the ESCAPE trial identified that optimising the haemodynamic parameters such as cardiac output without modifying the above neurohumoral maladaptive response does not improve renal function or long-term outcomes. ${ }^{19}$ It argued that diminished forward flow alone (reduced cardiac output) does not account for the development of CKD and AKI in decompensated heart failure. ${ }^{19}$ More recent evidence has suggested that venous congestion (with resultant elevation in renal venous pressure), more than reduced cardiac output, appears to be a significant contributor to the development of CRS. ${ }^{19-21}$ It is worth mentioning that diuretics use, especially with intravenous administration, can theoretically result in further deterioration in glomerular filtration. This is mainly due to increased SNS and RAAS activity in response to diuretics induced reduction in intraglomerular pressure.

\section{Approach to management}

\section{Decongestion}

The primary target for treatment of patients with decompensated heart failure is to relieve the symptoms and adverse haemodynamic effects of congestion. This requires controlled but effective diuresis, and will generally require a significant step up in loop diuretic dose (as compared to pre-admission) with intravenous regimen. ${ }^{20}$

Although inotropic and vasoactive agents that target cardiac output and peripheral resistance are occasionally employed to augment diuresis, they come at a cost with increased myocardial workload and potential to cause excess mortality. ${ }^{21}$

Diuretic resistance, especially in advanced CRS, is a huge challenge. ${ }^{22}$ There are many proposed causes of diuretic resistance including rebound sodium reabsorption as the diuretic effects wears off, excess sodium intake, remodelling of the thiazide and mineralocorticoid receptors resulting in increased sodium reabsorption downstream in the nephron, reduced tubular secretion of loop diuretics due to competitive inhibition of the transporter by elevated levels of endogenous organic acids in renal impairment, reduced protein binding of loop diuretics due to hypoalbuminaemia and metabolic acidosis. ${ }^{23}$ In practical terms, the key strategy is to ensure regular review of the patient's progress and in the absence of adequate diuresis (often judged by fluid balance or weight loss) a step up in diuretic therapy is required. Higher than normal doses of loop diuretics, frequent dosing or continuous infusion, with or without the addition of a thiazide diuretic and/or MRA, may therefore be necessary in order to overcome diuretic resistance. ${ }^{22,23}$ Sadly, patients with CRS are less likely to receive optimal doses of diuretics and RAASi. This is especially true if there is a rise in creatinine (stage $1 \mathrm{AKI}$ ) following initiation of the above therapy, but such renal functional change should not be considered as detrimental. Indeed, in the DOSE trial, worsening renal function in the high-dose furosemide group was transient, with no difference between the two groups in serum creatinine over the 60 day follow-up period and, in the UNLOAD trial, a reduction in the rate of hospitalisation for heart failure was noted to occur in association with a trend toward worsening renal function and greater weight loss in the ultrafiltration (UF) group. ${ }^{24,25}$ This evidence from clinical trials suggest that fears about the detrimental effects of deteriorating renal function in this clinical scenario may be unfounded. ${ }^{26}$ 
This is supported by studies demonstrating that congestion at discharge, irrespective of in hospital AKI, confers a worse prognosis than in those patients who have been adequately off-loaded of fluid even if they do have AKI at discharge. ${ }^{14}$

The use of UF in patients with acute decompensated heart failure (ADHF) held promise as a key method of decongestion after the UNLOAD trial showed improved outcomes and fewer readmissions with UF. ${ }^{25}$ However, UF as a regular treatment fell out of favour after the CARESS-HF study showed a higher rise in serum creatinine $(20.3 \mu \mathrm{mol} / \mathrm{L}$ vs $-3.5 \mu \mathrm{mol} / \mathrm{L} ; \mathrm{p}=0.003)$ without a significant difference in weight reduction $(12.6 \mathrm{~kg}$ vs $12.1 \mathrm{~kg} ; \mathrm{p}=0.58$ ) or symptoms in the UF group compared to the pharmacologic therapy group. In addition, the UF group was associated with higher rates of adverse events ( $72 \%$ vs $57 \%$; $p=0.03) .{ }^{27}$ Critics have highlighted the possible reasons for the difference in outcome between the two trials. The CARESS-HF trial optimised the diuretic treatment by using a protocol-driven high dose diuretic strategy which may have been more effective than the conservative diuretic strategy used in the UNLOAD trial. Furthermore, the patient populations within the two trials differed in that CARESS-HF was restricted to patients with renal impairment while the UNLOAD trial was not. Nevertheless, the use of UF for decongestion may still have its place in patients with refractory diuretic resistant $\mathrm{ADHF}$.

In summary, the key goal of therapy in decompensated heart failure is to eliminate fluid retention even if this leads to asymptomatic mild to moderate reductions in renal function or blood pressure.

\section{What to do with RAASi in acute kidney injury?}

Patients with acute decompensated heart failure are at high risk and several registries have demonstrated the in-hospital mortality rate to be between $4-11 \% .{ }^{28,29}$ Congestion is the main driver for the patient's symptoms leading to admission, and likely drives AKI seen in these overloaded patients. ${ }^{30}$ Decongestion with diuretics is crucial for symptom relief. ${ }^{31}$
Uncertainty can arise on how to manage the co-prescription of RAASi in the patient with CHF and 'worsening renal function'. This has been exacerbated by widespread inappropriate use of terms such as 'acute kidney injury' or 'nephrotoxic drugs' when referring to RAASi. In patients with $\mathrm{CHF}$, the change in renal function commonly reflects changes in renal haemodynamics as opposed to tubular injury. A recent study showed that intensive decongestion in patients with pre-existing AKI and ADHF can be associated with a rise in tubular injury biomarkers ( $\mathrm{N}$-acetyl b-D-glucosaminidase (NAG), kidney injury molecule-1 (KIM-1) and neutrophil gelatinaseassociated lipocalin (NGAL)). ${ }^{32}$ However, it was reassuring that recovery of renal function and fluid status were superior in those patients exhibiting increased tubular injury markers.

Guidance on how to address renal function changes has been confusing and unclear, probably because there has been little evidence to formulate the guidelines. The National Institute for Health and Care Excellence (NICE) AKI and CKD guidelines have advised ACEi or ARB dose reduction or even stopping these agents if serum creatinine rises by $>30 \%$ without an alternative explanation. ${ }^{33,34}$ The NICE heart failure guidelines reference the NICE CKD guidelines, whereas the European Society of Cardiology heart failure guidelines suggest that a $50 \%$ increase in creatinine should be the cut-off point for reducing or discontinuing RAASi therapy. ${ }^{35,36}$

This has led the Renal Association and the British Society for Heart Failure to collaborate to describe the interactions between heart failure, RAASi and renal dysfunction and they now have provided clear consensus guidance on the use of RAASi (including sacubitril valsartan) in patients with heart failure. ${ }^{37}$ For a flow chart for management based on the patient's presenting problem see Fig 1.

The first important part of deciding what to do with RAASi, in the setting of decompensation, is to assess its indication (prognostic benefit in $\mathrm{HFrEF}$ ) and the volume status of the patient to give context to the creatinine (and eGFR) results. The volume overloaded patient with raised jugular venous pressure, pulmonary/peripheral oedema and without hypotension needs diuretics for decongestion and RAASi should be continued unless there are marked changes in renal function.
Fig 1. Flow chart for management of a patient on renin-angiotensinaldosterone system inhibitors based on the patient's presenting problem. $\mathrm{ACE}$ = angiotensin converting enzyme inhibitor; AKI = acute kidney injury; ARB $=$ angiotensin receptor blocker; $\mathrm{BP}=$ blood pressure; IV = intravenous; MRA $=$ mineralocorticoid receptor antagonist; RAASi $=$ renin-angiotensin-aldosterone system inhibitors.

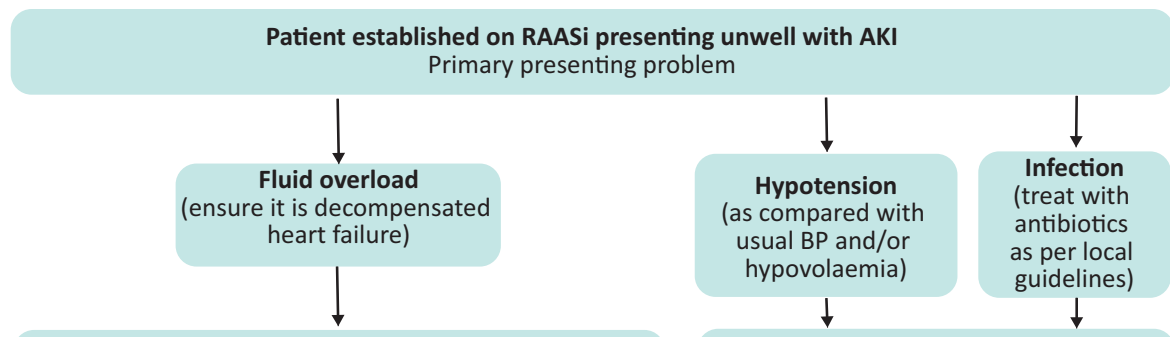

All decompensated heart failure:
- decongestion with diuretics
- often need high-dose IV loop diuretic - at least
double usual oral dose
- if using infusion, require bolus first
- may need combination with thiazide and MRA
HFrEF

Stop RAASi if not for prognostic benefit - May need IV fluids with careful and repeated clinical and biochemical review - May temporarily reduce or withhold RAASi even if for prognostic benefit eg if symptomatic hypotension despite volume correction, moderate-severe hyperkalaemia or progressive AKI 
Table 1. Recommendations for renin-angiotension-aldosterone system inhibitors based on creatinine change ${ }^{37}$

Change in renal function compared with
baseline

Increase in serum creatinine by $<30 \%$

Increase in serum creatinine by $30-50 \%$

Increase in serum creatinine by $>50 \%$

Severe renal dysfunction, for example, eGFR $<20 \mathrm{~mL} / \mathrm{min} / 1.73 \mathrm{~m}^{2}$

\section{HFpEF (assuming no other} prognostic indication)

Consider stopping ACEi/ARB/ARNi

Review MRA according to fluid status

Stop RAASi

Stop RAASi

Stop RAASi

\section{HFrEF}

Continue unless symptomatic hypotension

Consider reducing dose or temporary withdrawal ${ }^{a}$

Temporarily stop RAASi ${ }^{a}$

Stop RAASi if symptomatic uraemia irrespective of baseline function

${ }^{a}=$ Re-initiate and/or re-titrate when renal function improved in patients with $\mathrm{HFrEF} ; \mathrm{ACEi}=$ angiotensin converting enzyme inhibitor; $\mathrm{ARNi}=$ angiotensin receptor neprilysin inhibitor; $\mathrm{ARB}$ = angiotensin receptor blocker; $\mathrm{eGFR}=$ estimate glomerular filtration rate; $\mathrm{HFpEF}$ = heart failure and preserved ejection fraction; $\mathrm{HFrEF}=$ heart failure with reduced ejection fraction; MRA = mineralocorticoid receptor antagonist; RAASi = renin-angiotension-aldosterone system inhibitors. Reproduced with permission from Clark AL, Kalra PR, Petrie MC et al. Change in renal function associated with drug treatment in heart failure: national guidance. Heart 2019;105:904-10.

See Table 1 for a summary of management based on whether the patient has reduced or preserved (no clear prognostic benefit of RAASi) left ventricular function and considering the level of creatinine increase. ${ }^{37}$

If RAASi do need to be reduced or discontinued in patients with HFrEF it is vital to repeat clinical and biochemical assessment with a view to reinitiate and/or re-up-titrate RAASi when renal function has improved, as there is prognostic benefit, and there are potential deleterious effects in those patients who are left off them.

Once clinical assessment shows the patient has been decongested, a disproportionate increase in the blood urea compared to the creatinine suggests that the diuretics can be reduced at this point.

Symptomatic hypotension should prompt withdrawal of other anti-hypertensives such as calcium-channel blockers in the first instance. If the patient remains hypotensive with symptoms and an increasing creatinine, then two considerations are important.

$>$ Is the congestion now resolved? Fluid status should be rechecked, RAASi reduced or omitted and if the patient is considered euvolaemic diuretic doses can be reduced.

> Is the patient in cardiogenic shock with pulmonary oedema? This situation demands urgent cardiologist / high-level care input.

\section{Role of heart failure specialist team}

A multidisciplinary approach, especially involving heart failure specialist nurses, is fundamental to improving the outcomes of patients with $\mathrm{CHF}$ both during hospitalisation and following discharge. ${ }^{38}$ It is a key recommendation in the NICE CHF guidelines. ${ }^{35}$ Where the patients are looked after also seems to matter - patients receiving care on a cardiology ward by a bespoke specialist team have a lower chance of in-hospital mortality (6.5\%) compared to the $12.6 \%$ annual mortality of those that are not, although there may be other cofounders that affect these statistics (Fig 2). Even if patients are not managed on a cardiology ward, specialist input (in-reach) is associated with better survival and a major factor is that patients are more likely to have been started and continued on prognostically significant disease modifying drugs. Those discharged on all three of the main classes of disease-modifying drugs (ACEi or ARB, beta blockers and MRA) had a 1 -year mortality rate of $15 \%$ compared to $30 \%$ for those leaving hospital without any of these three key drug classes. ${ }^{6}$

\section{Follow-up}

For all patients hospitalised with heart failure, comprehensive discharge planning and post-discharge support is vital and can significantly reduce readmission rates. ${ }^{39}$ This includes early (within 2 weeks) post-discharge review by heart failure specialist nurse teams either based in the community or secondary care. ${ }^{40}$ The latter is a crucial assessment in which fluid balance and renal function can be assessed and treatment adjusted. If patients have had RAASi stopped or down-titrated during the hospitalisation period, then attempts should be made to re-optimise these drugs.

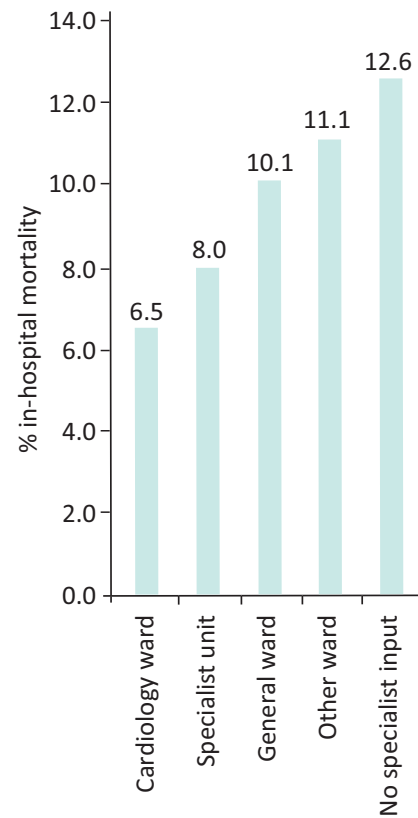

Fig 2. Patient chances of in-hospital mortality based on location of care. 


\section{References}

1 Conrad N, Judge A, Tran J et al. Temporal trends and patterns in heart failure incidence: a population-based study of 4 million individuals. Lancet 2018;391:572-80.

2 Bottle A, Kim D, Aylin P et al. Routes to diagnosis of heart failure: observational study using linked data in England. Heart 2018;104:600-5.

3 Burnett $\mathrm{H}$, Earley A, Voors AA et al. Thirty years of evidence on the efficacy of drug treatments for chronic heart failure with reduced ejection fraction. Circ Heart Fail 2017;10:e003529.

4 McMurray J], Packer M, Desai AS et al. Angiotensin-neprilysin inhibition versus enalapril in heart failure. $N$ Engl J Med 2014; 371:993-1004.

5 Solomon SD, McMurray JJV, Anand IS et al. Angiotensin-neprilysin inhibition in heart failure with preserved ejection fraction. $N$ Engl J Med 2019;381:1609-20.

6 National Cardiac Audit Programme. National heart failure audit: 2016/17 summary report. NCAP, 2018. www.nicor.org.uk/nationalcardiac-audit-programme

7 Hillege HL, Girbes ARJ, de Kam PJ et al. Renal function, neurohormonal activation and survival in patients with chronic heart failure. Circulation 2000;102:203-10.

8 Damman K, Testani JM. The kidney in heart failure: an update. Eur Heart ] 2015;36:1437-44.

9 Forman DE, Butler J, Wang $Y$ et al. Incidence, predictors at admission, and impact of worsening renal function among patients hospitalized with heart failure. J Am Coll Cardiol 2004;43:61-7.

10 Rastogi A, Fonarow GC. The cardiorenal connection in heart failure. Curr Cardiol Rep 2008;10:190-7.

11 Heywood JT, Fonarow GC, Costanzo MR et al. High prevalence of renal dysfunction and its impact on outcome in 118,465 patients hospitalized with acute decompensated heart failure: a report from the ADHERE database. J Card Fail 2007;13:422-30.

12 Hillege HL, Nitsch D, Pfeffer MA et al. Renal function as a predictor of outcome in a broad spectrum of patients with heart failure. Circulation 2006;113:671-8.

13 Damman K, Valente MAE, Voors AA et al. Renal impairment, worsening renal function, and outcome in patients with heart failure: an updated meta-analysis. Eur Heart ] 2014;35:455-69.

14 Fudim M, Loungani R, Doerfler SM et al. Worsening renal function during decongestion among patients hospitalized for heart failure: Findings from the Evaluation Study of Congestive Heart Failure and Pulmonary Artery Catheterization Effectiveness (ESCAPE) trial. Am Heart ] 2018;204:163-73.

15 Turner N, Lamiere N, Goldsmith D et al (eds), Oxford textbook of clinical nephrology, 4th edn. Oxford: Oxford University Press, 2015.

16 Boerrigter G, Burnett JC. Cardiorenal syndrome in decompensated heart failure: prognostic and therapeutic implications. Curr Heart Fail Rep 2004;1:113-20.

17 Kumar U, Wettersten N, Garimella PS. Cardiorenal syndrome: Pathophysiology. Cardiol Clin 2019;37:251-65.

18 Ronco C, Cicoira M, McCullough PA. Cardiorenal syndrome type 1: pathophysiological crosstalk leading to combined heart and kidney dysfunction in the setting of acutely decompensated heart failure. J Am Coll Cardiol 2012;60:1031-42.

19 Nohria A, Hasselblad V, Stebbins A et al. Cardiorenal interactions: insights from the ESCAPE trial. J Am Coll Cardiol 2008;51:1268-74.

20 Rubinstein J, Sanford D. Treatment of cardiorenal syndrome. Cardiol Clin 2019;37:267-73.

21 Hatamizadeh P, Fonarow GC, Budoff M] et al. Cardiorenal syndrome: Pathophysiology and potential targets for clinical management. Nat Rev Nephrol 2013;9:99-111.

22 Rangaswami J, Bhalla V, Blair JEA et al. Cardiorenal syndrome: Classification, pathophysiology, diagnosis, and treatment strategies: a scientific statement from the American Heart Association. Circulation 2019;139:e840-78.
23 De Bruyne LKM. Mechanisms and management of diuretic resistance in congestive heart failure. Postgrad Med J 2003;79:268.

24 Lala A, McNulty SE, Mentz RJ et al. Relief and recurrence of congestion during and after hospitalization for acute heart failure: Insights from Diuretic Optimization Strategy Evaluation in Acute Decompensated Heart Failure (DOSE-AHF) and Cardiorenal Rescue Study in Acute Decompensated Heart Failure (CARESS-HF). Circ Heart Fail 2015;8:741-8.

25 Costanzo MR, Guglin ME, Saltzberg MT et al. Ultrafiltration versus intravenous diuretics for patients hospitalized for acute decompensated heart failure. J Am Coll Cardiol 2007;49:675-83.

26 Valika AA, Gheorghiade M. ACE inhibitor therapy for heart failure in patients with impaired renal function: a review of the literature. Heart Fail Rev 2013;18:135-40.

27 Bart BA, Goldsmith SR, Lee KL et al. Ultrafiltration in decompensated heart failure with cardiorenal syndrome. N Engl J Med 2012;367:2296-304.

28 Follath F, Yilmaz MB, Delgado JF et al. Clinical presentation, management and outcomes in the Acute Heart Failure Global Survey of Standard Treatment (ALARM-HF). Intensive Care Med 2011;37:619-26.

29 Adams KF Jr, Fonarow GC, Emerman CL et al. Characteristics and outcomes of patients hospitalized for heart failure in the United States: rationale, design, and preliminary observations from the first 100,000 cases in the Acute Decompensated Heart Failure National Registry (ADHERE). Am Heart J 2005;149:209-16.

30 Damman K, Navis G, Smilde T et al. Decreased cardiac output, venous congestion and the association with renal impairment in patients with cardiac dysfunction. Eur J Heart Fail 2007;9:872-8.

31 Mullens W, Abrahams Z, Francis GS et al. Importance of venous congestion for worsening of renal function in advanced decompensated heart failure. J Am Coll Cardiol 2009;53:589-96.

32 Rao VS, Ahmad T, Brisco-Bacik M et al. Renal effects of intensive volume removal in heart failure patients with pre-existing worsening renal function. Circ Heart Fail 2019;12:1-11.

33 National Institute for Health and Care Excellence. Acute kidney injury: prevention, detection and management. Clinical guideline [CG169]. London: NICE, 2013.

34 National Institute for Health and Care Excellence. Chronic kidney disease in adults: assessment and management. Clinical guideline [CG182]. London: NICE, 2014.

35 National Institute for Health and Care Excellence. Chronic heart failure in adults: diagnosis and management. NICE guideline [NG106]. London: NICE, 2018.

36 Ponikowski P, Voors AA. 2016 ESC Guidelines for the diagnosis and treatment of acute and chronic heart failure: The task force for the diagnosis and treatment of acute and chronic heart failure of the European Society of Cardiology (ESC). Eur Heart J 2016;37:2129.

37 Clark AL, Kalra PR, Petrie MC et al. Change in renal function associated with drug treatment in heart failure: national guidance. Heart 2019;105:904-10.

38 Finlay A. Multidisciplinary strategies for the management of heart failure patients at high risk for admission A systematic review of randomized trials. J Am Coll Cardiol 2004:44:810-9.

39 Phillips CO, Wright SM, Kern DE et al. Comprehensive discharge planning with post discharge support for older patients with congestive heart failure: a meta-analysis. JAMA 2004;291:1358-67.

40 British Heart Foundation. Heart failure nurse services in England: Executive summary. London: BHF, 2008. www.wales.nhs.uk/ sitesplus/documents/986/BHF $\% 2$ Heart $\%$ 20Failure $\% 20$ Nurse $\% 20$ Evaluation \% 20in \% 20England \% 20-\% 20Exec\% 20Summary.pdf

Address for correspondence: Prof Philip Kalra, Salford Royal NHS Foundation Trust, Stott Lane, Salford M6 8HD, UK. Email: philip.kalra@srft.nhs.uk 